\title{
COMO DESENVOLVER PROJETOS DE ATENÇÃO À SAÚDE MENTAL NO PROGRAMA DE SAÚDE DA FAMÍLIA
}

\author{
HOW TO DEVELOP PROJECTS OF ATTENTION TOWARDS MENTAL HEALTH INSIDE THE PROGRAM OF \\ FAMILY HEALTH \\ COMO DESARROLLAR PROYECTOS DE ATENCIÓN PARA LA SALUD MENTAL EN EL PROGRAMA DE SA- \\ LUD DE LA FAMILIA
}

\section{Tânia Mara Xavier SCÓZ ${ }^{1}$ Rosangela Maria FENILI ${ }^{2}$}

RESUMO: O desenvolvimento de Projetos de Atenção à Saúde Mental no Programa de Saúde da Família (PSF) é apresentado a partir de um resgate sucinto de alguns dos aspectos que compõem o PSF, a Reforma Psiquiátri$\mathrm{ca} /$ Saúde Mental e o Sistema Único de Saúde. A inserção de ações de atenção a saúde mental no PSF, a partir, da existência e inexistência de um dos serviços substitutivos da rede de saúde mental nos municípios, aponta algumas possibilidades de articulação e desenvolvimento conjunto do como fazer.

PALAVRAS CHAVE: Saúde mental, Programa de saúde da família

ABSTRAT: The development of projects of attention towards mental health inside the Program of Family Health (PFH) is presented based on a brief rescue of some aspects that compose the PFH, the Psychiatric Reform/Mental Health and the Unique Heath System. The insertion of attentive actions towards mental health in the PFH from the existence and the non-existence of one of the substitutives services of the mental health net around the cities, points out some articulation possibilities and an united development of how to do it.

KEY-WORDS: Mental Health, Program of Family Health

RESUMEN: El desarrollo de Proyectos de Atención para la Salud Mental en el Programa de Salud de la Familia (PSF) se presenta a partir de un rescate suscinto de algunos de los aspectos que componen el PSF, la Reforma Psiquiátrica/Salud Mental y el Sistema Único de Salud. La inserción de acciones de atención a la salud mental en el PSF, a partir, de la existencia e inexistencia de uno de los servicios sustitutos de la red de salud mental en las Municipalidades, señalan algunas posibilidades de articulación y desarrollo conjunto de como hacerse.

PALABRAS CLAVES: Salud Mental, Programa de Salud de la Familia.

\section{INTRODUÇÃO}

Como desenvolver Projetos de Atenção à Saúde Mental no Programa de Saúde da Família (PSF), no momento em que a saúde mental passa por um processo altamente dinâmico de reformulações e busca da sua própria estruturação? Como conciliar temas tão intrinsecamente ligados e tão conflitantemente capazes de despertar nossa capacidade de sonhar em um futuro tão brilhantemente próximo e tão assustadoramente desafiador? Como transformar um saber, um fazer, se estamos tão rotineiramente acostumados com as relações saúde/doença nos seus aspectos causa e efeito, com o viver e morrer como um processo natural evolutivo de nossa espécie, com a riqueza e a pobreza tão visivelmente evi- denciadas e tão pouco constrangedoras, com a desigualdade social cada vez mais exacerbada e distante de uma prática solidária, humanitária que atenda as reais necessidades do cidadão? Como, como, como....?

Acreditamos que o como não se constrói sozinho. Conjuntamente outras interrogações, o que, por que, para que, para quem, onde, quem, surgem em nossas mentes e nos remetem a necessidade de buscar subsídios que apontam para o entendimento do como fazer nas suas mais diversas formas. Assim, pensamos ser necessário resgatar sucintamente alguns dos aspectos relacionados com o PSF, Reforma Psiquiátrica/Saúde Mental, principalmente na variável comum entre eles, ou seja, o atendimento do indivíduo no seu contexto

\footnotetext{
${ }^{1}$ Enfermeira, Mestre em Ergonomia

${ }^{2}$ Enfermeira, Docente do Departamento de Enfermagem da Universidade Federal de Santa Catarina, Mestre em Assistência de Enfermagem. Rua Eugênio Raulino Koerich -619, Bloco E- Ap. 201 - Campinas - São José - SC CEP - 88101-060 - Telefone - (048) 2412624 - E-mail - fenili@nfr.ufsc.br
} 
familiar de acordo com os preceitos do Sistema Único de Saúde (SUS) e o grau de complexidade do atual serviço de saúde.

\section{ASPECTOS PONTUAIS DO SUS / PSF / REFORMA PSIQUIÁTRICA}

\section{SISTEMA ÚNICO DE SAÚDE}

(SUS) : Com o objetivo de reorientar o sistema de saúde brasileiro, é implantado o SUS, que visa através das várias instituições dos três níveis do governo (união, estados e municípios), e do setor privado contratado e conveniado dar cumprimento ao mandamento constitucional de disciplinar legalmente a proteção e a defesa da saúde.

Dentre os princípios que norteiam SUS destacam-se: o atendimento gratuito a todos, sendo o indivíduo e a comunidade, no qual está inserido, assistido de forma integral, garantindo-lhes o respeito a dignidade humana. A execução das ações deve ser descentralizada, delegando a responsabilidade para os municípios, os estados e a união de acordo com a abrangência do problema. O princípio da racionalidade, ou seja, o oferecimento de ações e serviços de acordo com as necessidades da população, deve ocorrer de maneira a atender a eficiência e eficácia do SUS, garantindo assim a qualidade da assistência. Através dos Conselhos de Saúde (nacional, estaduais e municipais), o exercício do controle social do SUS, busca assegurar o aspecto democrático, isto é, o direito de participação de todos os seguimentos envolvidos com o sistema. As desigualdades existentes devem direcionar as ações do sistema, ajustando-as as necessidades de cada parcela da população, respeitando o princípio da equanimidade (SC, 2001).

\section{PROGRAMA (ESTRATÉGIA) DE} SAÚDE DA FAMÍLIA (PSF) : Em dezembro de 1993 o Ministério da Saúde (MS) cria um grupo de trabalho com o objetivo de discutir a proposta de implantação de um modelo de saúde mais adequado as necessidades do país. Como resultado o grupo aponta para a criação de um programa que elege o núcleo familiar como foco de suas ações, ou seja, o PSF, lançado no início de 1994 pelo MS. Ao eleger a família como um subsistema decisório, consumidor e parceiro, verifica-se a incorporação dos princípios básicos do SUS, inserindo a unidade de saúde da família no primeiro nível de ações e serviços do sistema local de assistência, denominado atenção básica.

De acordo com Santos et al. (2000,

A importância de visualizar a família, como uma unidade de cuidado, é expressada, freqüentemente, referindo que 'o paciente não é sozinho, ele vem de algum lugar e se trabalha a valorização das pessoas cuidadoras de quem necessita o cuidado quer sejam as figuras parentais e/ou outros profissionais' .

Os princípios da promoção da saúde, através do fortalecimento da atenção básica, tendo o PSF como seu eixo estruturante, permitem a construção da saúde através de uma troca solidária, crítica, capaz de fortalecer a participação comunitária, o desenvolvimento de habilidades pessoais, a criação de ambientes saudáveis e a reorganização de serviços de saúde, entre outros.

A visão sistêmica e integral do indivíduo em seu contexto familiar e social, é proporcionado através do trabalho "com as reais necessidades locais, por meio de uma prática apropriada, humanizada e tecnicamente competente, sincronizando o saber popular com o saber técnico científico, em um verdadeiro encontro de gente cuidando de gente" (SOUZA, 2000, p. 27).

Ainda para a mesma autora, a identificação de fatores de riscos e a busca de uma intervenção resolutiva é realizada através da vinculação da equipe de saúde multidisciplinar com a população, através de uma prática sustentada pela humanização e a saúde como um direito de cidadania, favorecendo a manifestação de qualidade de vida.

3. REFORMA PSIQUIÁTRICA: Conforme Serrano (1998, p. xix), a reforma psiquiátrica "é o processo político assistencial, em nível mundial e em diferentes formas, 
derivado da queda do paradigma asilar, que deixa de ser hegemônico, no tratamento psiquiátrico, em discussão desde a década de 60", e que no Brasil reúne a reformulação da assistência, mudanças na cultura e mudanças normativas e jurídicas.

O processo de desinstitucionalização teve início nos anos 80 com o desenvolvimento de uma política em favor da saúde mental, e tendo como marco de referência a desinstitucionalização italiana. Ele se firma a partir do Sistema Único de Saúde, e é referendado com a realização de duas Conferências de Saúde Mental, a primeira no Rio de Janeiro, em 1987, outra em Brasília, em 1992 (FENILI, 1999, p. 38).

A desinstitucionalização procura preservar a subjetividade do cliente, sua história de vida, suas relações interpessoais, estabelecendo formas de tratamento em serviços abertos, criativos e maleáveis que atendam individualmente às suas necessidades e que o sustente no seu meio social (MORENO, 1995).

Em decorrência de todo o movimento político que discutia o modelo no qual estava inserida a saúde mental, surge no plano jurídico o Projeto de Lei da Câmara $\mathrm{n}^{\circ} 8$ de $1991, n^{\circ} 3.657$ de 1989 , na casa de origem de autoria do Deputado Paulo Delgado, como substitutivo ao Decreto $n^{\circ} 24.559$, de 03 de julho de 1934, que Dispõe sobre a extinção progressiva dos manicômios e sua substituição por outros recursos assistenciais e regulamenta a internação compulsória (BRASIL). Essa lei vem "respaldar a desinstitucionalização, como uma estratégia alternativa ao modelo de saúde mental vigente" (SADIGURSKY, 1997, p. 29). Mas somente em 06 de abril de 2001 foi aprovado pelo presidente da República como a Lei $\mathrm{N}^{\circ}$ 10.216. O combate aos estigmas é a principal medida para garantir o sucesso da Lei da Reforma Psiquiátrica. Essa lei segue a diretriz da OMS: garantir tratamento aos pacientes sem necessidade de inteEraçembro de 2001 realiza-se a III Conferência de Saúde Mental, em Brasília, tendo como título, proposto pela Organização Mundial de Saúde, "Cuidar sim, excluir não". Baseada na Lei aprovada procurou direcionar suas discussões, em três temáticas: reorientação do modelo assistencial; recursos humanos e financiamento; controle social, acessibilidade, direitos e cidadania.

4. REFORMA SUBSTITUTIVA: Reforma substitutiva é todo tipo de ação que visa apresentar novas formas de cuidado da pessoa no seu processo de vida, através da reorientação do modelo assistencial.

Em se tratando de PSF e Reforma Psiquiátrica acredita-se que, a efetiva meIhora na qualidade de vida converge para a realização do cuidado em saúde no próprio núcleo familiar.

A substituição do modelo hospitalocêntrico, centrado na doença, na exclusão social, intramuros, por um novo modelo de atenção descentralizado e de base comunitária, exigirá também uma adaptação dos profissionais, que deverão, através do processo de capacitação, se instrumentalizarem para permitir a viabilização do novo modelo assistencial. Concomitante, o grupo familiar parceiro deste modelo, deve assumir seu papel de ajudar o membro mais fragilizado a conviver com a situação, desta forma diminuindo a internação.

Apesar da legislação própria que determina a especificidade de suas ações, existem princípios que possibilitam a convergência de ações de saúde mental no PSF, por exemplo:

1. Princípios do SUS, apontados anteriormente;

2. Territorialização e responsabilidade pela demanda ;

3. Desinstitucionalização das pessoas internadas e a construção de rede de cuidado capaz de atender a demanda;

4. Modalidades de cuidado que garantam a escuta, o vínculo, que potencializem as singularidades, as biografias, as raízes culturais, as redes de pertencimento e estejam atentas e recusem as formas de medicalização, psiquiatrização e psicologização;

5. Planejamento, desenvolvimento e avaliação das ações em equipe multiprofissional; 
6. Desenvolvimento das ações de saúde mental nas unidades de saúde, nos domicílios, nos lugares e recursos comunitários;

7. Atendimento às pessoas em situações de gravidade, através de ações efetivas que viabilizem o acesso.

\section{PROJETO DE ATENÇÃO A SAÚDE MEN- TAL NO PSF}

A intervenção conjunta da equipe em saúde mental e o PSF tem como estratégia atingir a meta de substituição do confinamento nos hospitais psiquiátricos pelo cuidado comunitário das pessoas que sofrem com transtornos mentais (SILVA et al., 200_ ) .

Para subsidiar a resposta do como elaborar Projeto de Atenção a Saúde Mental no PSF, faz-se necessário conhecer a maneira como os PSF vêm desenvolvendo suas ações junto a comunidade. $O$ relato de algumas experiências apontam para a realização de ações de saúde mental em alguns PSFs, bem como a provável inexistência em outros.

Frente a isso apresenta-se uma proposta do como inserir ações de atenção a saúde mental no PSF, considerando-se as possibilidades de, existência de um dos serviços substitutivos da rede de saúde mental no município desvinculada do PSF, e a ausência deste.

Entende-se por rede de saúde mental o conjunto de ações concebidas a partir da Reforma psiquiátrica, ou seja, a formação de serviços substitutivos organizados em nível de complexidade capaz de atender a demanda da população. Neste contexto destaca-se a hierarquização da rede de atenção em saúde mental constituída pela Atenção Básica, Centro de Atenção Psicossocial (CAPS) Simplificado, Núcleo de Atenção Psicossocial (NAPS)/ CAPS de média complexidade, Serviços residenciais terapêuticos, Hospital dia, Leitos psiquiátricos em hospital geral, Serviço de urgência psiquiátrica em hospital geral e Hospital psiquiátrico (BRASIL, 2001).
Salienta-se a importância da comunhão de esforços entre PSF e serviços substitutivos, pois acredita-se como Lancetti (BRASIL, 2000, p.46) que a saúde mental é essencial ao PSF porque a doença mental não ocorre separada da saúde física. Outro fato é a singularização do atendimento no PSF; o paciente deixa de ser um prontuário para se transformar numa pessoa conhecida, obrigando as equipes de trabalho a lidar com o sofrimento humano, uma difícil tarefa para a qual não estão preparadas.

\section{COMO FAZER QUANDO EXISTE SERVI- ÇOS/ REDE DE SAÚDE MENTAL?}

A existência do serviço/ rede de saúde mental e do PSF nos leva a considerar que o prioritário é o desenvolvimento de ações conjuntas, articuladas de acordo com o grau de complexidade que a situação exige, no atendimento as reais necessidades de saúde da família.

Dentro as possibilidades de articulação e desenvolvimento conjunto de ações destaca-se:

X capacitação e supervisão para as equipes de saúde da família (ESF) completa, desenvolvendo temáticas relacionadas aos direitos de cidadania e direitos humanos (Estatuto da criança e do adolescente - ECA, direito dos portadores de transtornos mentais, dos idosos, das mulheres, etc.); princípios da reforma psiquiátrica e do PSF; família, grupos e rede social; intervenção na crise; problemas relacionados ao álcool e outras drogas; sexualidade/DST/AIDS ; uso de psicofármacos, entre outros. Reforça-se a idéia de que a capacitação, enquanto educação continuada, se desenvolva reciprocamente entre as equipe do PSF e do serviço;

$X$ intervenções familiares comunitárias conjuntas como um instrumento de capacitação ( utilização da escuta como instrumento terapêutico na compreensão da dinâmica 
familiar e das relações sociais; oIhar atento e sensível dos pontos de vulnerabilidade de quebra dos vínculos familiares e sociais; estreitamento dos vínculos da equipe com os familiares portadores de transtornos psiquiátricos severos);

$x$ criação de uma equipe volante de saúde mental por parte dos serviços de saúde mental substitutivos, para o desenvolvimento das ações junto as ESF;

$x$ integrar a saúde mental nas diversas atividades de grupos oferecidas pelo PSF (caminhadas, ginástica terapêutica, salas de espera, oficinas);

$X$ articulações com as diversas formas de organizações populares (associações de bairro, grupos de auto-ajuda, etc), buscando construir novos espaços de reabilitação psicossocial ( oficinas comunitárias, hortas comunitárias, cooperativas, etc);

X promover ações para diminuir o comportamento violento, abuso de álcool/consumo de drogas, isolamento social/afetivo, entre outros, através da realização de palestras, debates, atividades artísticas e de grupos.

Para que essas ações sejam eficientes e eficazes acredita-se que existam duas formas de encaminhamento intrinsecamente ligadas a vontade política e técnica de resolutividade. A primeira, por iniciativa dos serviços substitutivos na área da saúde mental através das situações apontadas anteriormente e a outra realizada pela equipe do PSF através da busca constante e articulada com esses serviços, seja através de encaminhamentos, solicitação de supervisão, qualificação permanente e outros.

Os profissionais envolvidos no cumprimento destas ações devem ser conforme Lancetti (BRASIL,2000, p. 46) " pessoas corajosas com vontade de experimentar, pois vão atuar diretamente com a loucura, com a violência, sem proteção, sem muros, apenas com o corpo e a inteligência. Ape- nas uma orelha que escuta, uma pessoa com a capacidade de compreensão".

\section{COMO FAZER QUANDO NÃO EXISTE SERVIÇOS/ REDE DE SAÚDE MENTAL?}

Considera-se, como ponto de partida, a existência do PSF, a ausência de serviços de saúde mental estruturados e uma demanda de atenção, expressas por frases: familiar "pegar remédios de nervos", "falar de meus problemas com a psicóloga"; - da equipe "perder o medo de lidar com o doente mental", "saber lidar melhor com as minhas angústias e da comunidade".

Para a resolução desta problemática, a equipe do PSF poderá, solicitar a Secretaria de Saúde (níveis municipais, estaduais, federal) capacitação dos seus profissionais como requisito básico para o atendimento das demandas apontadas. Esta capacitação segundo Casé (200-, p. 123) deve envolver um planejamento no qual sejam "delineadas as seguintes ações: - pesquisa e estudo de campo em área piloto, - oficinas de sensibilização das equipes do PSF, - monitoração sistemática das ações, - elaboração de uma proposta de atenção a saúde mental".

Uma vez capacitada e incorporadas as ações de atenção a saúde mental, a equipe do PSF, então instrumentalizada, torna-se mais apta a identificação de situações e prestar os cuidados de atenção básicos a família portadora de sofrimento psíquico. Torna-se, também, mais evidente, o reconhecimento da limitação de sua ação, o que por sua vez poderá levar a necessidade de fortalecimento de busca de reforço de acordo com a referência existente em sua região.

Pode ainda ocorrer a sensibilização da equipe no sentido de buscar uma maior resolutividade das questões emergentes, através da contratação de profissionais que irão compor a equipe de saúde mental junto ao PSF. Esta equipe dará início a uma proposta de atenção a saúde mental que poderá ser o embrião da constituição da rede de atendimento básico de saúde mental.

Qualquer que seja a alternativa de implementação das ações de saúde mental 
no PSF, julga-se, imprescindível reforçar o papel evidente de uma ação conjunta, respeitando os princípios básicos de reorientação do modelo assistencial, em que a estratégia de desinstitucionalização, atenção biopsicossocial interdisciplinar e a ampla participação e o controle social dos usuários, permitam atender os preceitos maiores do SUS, PSF e Reforma psiquiátrica.

\section{CONSIDERAÇÕES FINAIS}

Como bem recordam Sampaio e Barroso (200-, p.184) "O dono e o cliente do sistema de saúde são a cidade e o cidadão", o que a nosso ver determina a relação de direitos e deveres quanto a responsabilidade pela eficácia e eficiência de tal sistema”.

A inclusão das ações de saúde mental no PSF dependem de uma política de saúde nacional, estadual e municipal que garanta formas de financiamento capaz de manter a integração e a operacionalização das estratégias de promoção, prevenção, tratamento e reinserção social, nestas modalidades de serviços substitutivos.

Dependem, também, da disponibilidade para a mudança da organização dos processos de trabalho. Considera-se que todas as pessoas envolvidas no planejamento, implantação, execução e avaliação da construção de uma rede de atenção básica de saúde estão constantemente no processo dinâmico do ensinar-aprender... ou seja, no processo contínuo de trocas em que o relacionamento interpessoal permita assistir, como bem diz Santos (2000, p.52), "a todos os homens e ao homem total".

O controle social efetivo da aplicação dos princípios SUS, PSF e Reforma psiquiátrica, realizadas, principalmente, através da participação ativa das comunidades, nos conselhos, associações entre outros, é uma das formas de garantia de continuidade no desenvolvimento das conquistas até então realizadas.

A compreensão dos vários níveis que compõe a complexidade do trabalho territorial, apontada pelas reformas substitutivas, garante o cuidado integral, tanto quanto, reafirma a responsabilidade de todos os envolvidos para a sua continuidade.

$\mathrm{Na}$ construção da saúde social para todos, a estratégia do PSF e da Reforma psiquiátrica, vêm se configurando numa forma inovadora de se fazer saúde, que fundamentada em uma nova ética social consolida a visão da família como a base, o centro de referência, o primeiro núcleo psicossocial no qual deve estar centrado a resolutividade das ações.

Como toda ação está imbuída de reflexão e, vice-versa, o término deste escrito objetiva contribuir para a sua ação/reflexão, através da leitura das transcrições a seguir:

- "A gente não pode mudar tudo, mas pode fazer tudo para mudar" (CAMINHA citada por SOUZA, 2000, p.27).

- "Intervir nas famílias é também intervir na ordem institucional alimentando a produção de uma nova subjetividade" (GONÇALVES, 200-, p. 116).

-"Sonhamos com uma revolução de saberes, fazeres e sentimentos, pois acreditamos ser este o único caminho possível" (CASÉ, 200-, p.134).

- "O desafio consiste em buscar e conquistar a cidadania dos pacientes com ações conjuntas das equipes de saúde, dos familiares e da comunidade " (CASÉ, 200p.135).

- "Os agentes comunitários de saúde são os grandes descobridores dos recursos escondidos da comunidade" (FRANCO; LANCETTI, 200-, p.107).

- "Nosso desafio é compreender o movimento familiar, acolhê-lo e continuar" (ROCHA; DAVID, 200-, p.92).

-"Eu vejo a vitória de um projeto onde todos podem contribuir com o que há de mais importante no ser humano que é solidariedade e o amor" (MATTOS, 200-, p.58).

\section{REFERÊNCIAS BIBLIOGRÁFICAS}

BRASIL. Senado Federal. Projeto de lei da Câmara $n^{\circ} 8$ de 1991. Mimeografado.

BRASIL, Ministério da Saúde. Orientações para organização da atenção aos portadores de transtornos mentais nos estados e municípios, de acordo com as di- 
retrizes NOAS-SUS 01/2001. Mimeografado.

BRASIL, Ministério da Saúde. Reorganização do sistema de saúde mental: uma experiência do Qualis2. Revista Promoção da Saúde. Ano 2, n.3, p. 446, ag.2000.

CASÉ, Vânia. Saúde mental e sua inteface como programa de saúde da família: quatro anos de experiência em Camaragibe. SaúdeLoucura 7, São Paulo, n. 1, p. 121-135, 200-.

FENILI, Rosangela Maria. Desvelando necessidade contrária através da assistência de Enfermagem ao cliente psiquiátrico. Florianópolis, 1999, 157 p. Dissertação de Mestrado em Assistência de Enfermagem - Curso de PósGraduação em Enfermagem, Universidade Federal de Santa Catarina.

FRANCO, Luiza, LANCETTI, Antônio. Quatro observações sobre as parcerias. SaúdeLoucura 7, São Paulo, n. 1, p. 103-107, 200-.

GONÇALVES, Paulo L. A unidade de referência e a equipe volante. SaúdeLoucura 7, São Paulo, n. 1, p. 109-116, 200-.

MATTOS, Solange Aparecida F. Entre a loucura e a liberdade: a experiência de uma agente comunitária de saúde. SaúdeLoucura 7, São Paulo, n. 1, p. 5358, 200-.

MORENO, Vania. O processo de desinstitucionalização e a reforma psiquiátrica: representações sociais entre profissionais e ocupacionais da enfermagem. Ribeirão Preto, 1995, 227 p. Dissertação de Mestrado em Enfermagem - Escola de Enfermagem de Ribeirão Preto, Universidade de São Paulo.

SADIGURSKI, Dora. Desinstitucionalização do doente mental: expectativas a família. Ribeirão Preto, 1997, 224 p. Tese Doutorado em Enfermagem - Escola de Enfermagem de Ribeirão Preto, Universidade de São Paulo.

SANTA CATARINA. Secretaria de Estado da Saúde. Legislação Básica. SUS Sistema Único de Saúde. 2001.
SANTOS, Beatriz R. L. et al. Formando o enfermeiro para o cuidado à saúde da família: um olhar sobre o ensino de graduação. Revista Brasileira de Enfermagem, Brasília, v. 53, n. especial, p. 49-59, dez.2000.

SERRANO, Alan Indio. Onze estações e um devoto de São Jorge - discursos psicológicos num Serviço de Saúde. Florianópolis, 1988, 280 p. Dissertação Mestrado em Psicologia - Curso de Pós- Graduação em Psicologia, Universidade Federal de Santa Catarina.

SOPHIA, Daniela. Cuidar sim, excluir não. Revista Tema, Rio de Janeiro, n. 22, p.23-24, set.2001.

SOUSA, Maria de Fátima. A enfermagem reconstruindo sua prática; mais que uma conquista no PSF. Revista Brasileira de Enfermagem, Brasília, v. 53, n. especial, p. 25-30, dez.2000.

ROCHA, Sílvia R., DAVID, Maria do Rosário F. F. Um louco lúcido. SaúdeLoucura 7, São Paulo, n. 1, p. 85-92, 200-. 